\title{
A Unified Scaling Model in the Era of Big Data Analytics
}

\author{
Zhongwei Li \\ Univeristy of Texas at Arlington \\ Arlington, Texas \\ zhongwei.li@mavs.uta.edu
}

\author{
Feng Duan \\ Univeristy of Texas at Arlington \\ Arlington, Texas \\ feng.duan@mavs.uta.edu
}

\author{
Hao Che \\ Univeristy of Texas at Arlington \\ Arlington, Texas \\ hche@uta.edu
}

\begin{abstract}
As scale-out execution of big data analytics has become predominate datacenter workloads, it is of paramount importance to faithfully characterize the scaling properties for such workloads. To date, the most widely cited scaling laws for big data analytics is the traditional Amdahl's law, which was discovered well before the era of big data analytics. A key observation made in this paper is that both the system and workload models underlying the traditional scaling laws are too simplistic to fully characterize the scaling properties for big data analytics workloads. In this paper, we put forward a Unified Scaling model for Big data Analytics (USBA), based on a multi-stage system model and a discretized workload model. USBA allows for flexible workload scaling unifying the fixed-size and fixed-time workload models underlying Amdahl's and Gustafson's laws, respectively, and flexible system scaling in terms of both number of stages and degree of parallelism per stage. Moreover, to faithfully characterize the scaling properties for big data analytics workloads, USBA accounts for variabilities of task response times and barrier synchronization. Finally, application of USBA to the scaling analysis of four Sparkbased data mining and graph benchmarks demonstrates that USBA is able to adequately characterize the scaling design space and predict the scaling properties of real-world big data analytics workloads. This makes it possible to use USBA as a useful tool to facilitate job resource provisioning for big data analytics in datacenters.
\end{abstract}

\section{CCS CONCEPTS}

- General and reference $\rightarrow$ Performance; • Computing methodologies $\rightarrow$ Distributed computing methodologies;

\section{KEYWORDS}

big data analytics, performance modeling, MapReduce, Spark, Amdahl's Law, Gustafson's Law

\section{ACM Reference Format:}

Zhongwei Li, Feng Duan, and Hao Che. 2019. A Unified Scaling Model in the Era of Big Data Analytics. In Proceedings of (HP3C '19). ACM, New York, NY, USA, 11 pages. https://doi.org/10.1145/3318265.3318268

Permission to make digital or hard copies of all or part of this work for personal or classroom use is granted without fee provided that copies are not made or distributed for profit or commercial advantage and that copies bear this notice and the full citation on the first page. Copyrights for components of this work owned by others than ACM must be honored. Abstracting with credit is permitted. To copy otherwise, or republish, to post on servers or to redistribute to lists, requires prior specific permission and/or a fee. Request permissions from permissions@acm.org.

HP3C '19, March 8-10, 2019, Xi'an, China

(C) 2019 Association for Computing Machinery.

ACM ISBN 978-1-4503-6638-0/19/03...\$15.00

https://doi.org/10.1145/3318265.3318268

\section{INTRODUCTION}

Big data analytics are scale-out by design with a large number of data blocks to be processed in parallel. For such applications, a job may involve multiple stages of parallel task processing and task result exchanges for tasks mapped to up to tens of thousands of commodity servers in which the data blocks reside. Notable examples are data mining, machine learning, and graph processing based on, e.g., MapReduce [1], Spark [2], and/or Dryad [3] programming frameworks. As big data analytics have emerged as the predominant datacenter workloads and consumed a significant portion of datacenter resources, it is of paramount importance to gain a sound understanding of the scaling properties of such workloads, e.g., for job resource provisioning purpose.

To this end, one must establish good system and workload models for big data analytics workloads and then, on the basis of these models, predict the scaling behaviors for such workloads. Unfortunately, to date, no systematic approach of this kind has been developed. Consequently, the most widely cited scaling law for big data analytics, to date, is still the traditional, well-known Amdahl's law [4], which along with a seemingly competing, well-known scaling law Gustafson's law [5], were discovered well before the era of big data analytics. Since both the system and workload models underlying these scaling laws are too simplistic, applying these laws to the scaling analysis of big data analytics workloads often brings more confusion than clarity.

First, the underlying system model shared by Amdahl's and Gustafson's laws can be viewed as a two-stage system with a serial and a parallel execution stage. While the serial execution stage has one processing unit in it, handling the serial portion of the workload, the parallel execution stage involves a number of processing units processing the parallelizable portion of the workload in parallel. The workload models underlying both Amdahl's and Gustafson's laws assume that the size of the serial portion of the workload stays unchanged as the system scales out, which is justified, according to [5], as the serial portion of the workload normally corresponds to the program initialization phase that does not change as the problem size increases. This is in stark contrast to the system and workload structures for big data analytics, which in addition to the initialization phase, normally involves from a few to several thousands of parallel execution stages, which must be executed sequentially. Moreover, as the problem size increases, in general, the number of parallel execution stages may grow, effectively adding more parallel and serial workloads simultaneously.

Second, the workload models for the parallelizable portion of the workload underlying both Amdahl's and Gustafson's laws are too restrictive, i.e., the fixed-size workload model for Amdahl's law and the fixed-time workload model for Gustafson's law, for which the size of the parallelizable portion of the workload stays unchanged and scales linearly, respectively, as the system scales out. In contrast, 
big data analytics workloads may stay unchanged, scale linearly or sublinearly, depending on the resource availability with respect to the workload size (see Section 2 for more details).

In lieu of the lack of and the importance of gaining a sound and fundamental understanding of the scaling properties and to facilitate more effective job resource provisioning for big data analytics workloads, in this paper, we put forward a Unified Scaling model for Big data Analytics (USBA) that renders the traditional scaling models its special cases. It makes the following major contributions. First, USBA is a unified scaling model, from which the well-known Amdahl's and Gustafson's laws, and other useful performance bounds can be derived as special cases. Second, USBA is a general scaling model that allows for flexible multi-stage system and workload scalings; variability of task response times; and barrier synchronization. Hence it can be applied to the scaling analysis of real-world big data analytics workloads, in the presence of a wide range of system and workload scaling behaviors and variability of task response times, e.g., task stragglers. Third, in USBA, a regression model is developed, that can match the measured performance data and predict the performance scaling within $20 \%$ errors for all four benchmarks studied ${ }^{1}$. Finally, the ability to work in a large workload design space allows USBA to capture the true scaling properties of big data analytics workloads that largely deviate from those predicted by the Amdahl's and Gustafson's laws.

The remainder of the paper is organized as follows. Section 2 provides the background information to motivate the current work. Section 3 introduces USBA. Section 4 presents the results for the scaling analysis of USBA, and its application to several Spark henchmarks. Section 5 reviews the related work. Finally, Section 6 concludes the paper.

\section{BACKGROUND AND MOTIVATIONS}

In this section, we first analyze in detail the Amdahl's and Gustafson's laws and the corresponding system and workload models. Then we identify, from both system and workload modeling points of view, the fallacies of applying these laws to characterize the scaling properties of big data analytics workloads, which motivate the current work.

To capture the workload models underlying the existing wellknown scaling laws and to be consistent with the USBA scaling model to be defined later, we view the system model shared by Amdahl's and Gustafson's laws as depicted in Figure 1. It is composed of a single processing unit and a set of parallel processing units arranged in a two-stage pipeline, executing the serial and parallelizable portions of the workload, respectively. In this model, each black dot represents a base task or workload unit, which cannot be further divided and parallelized. There is only one base task at the serial stage, representing the serial portion of the workload. This discretization of the workload leads to discretized versions of the two laws, as we shall see shortly.

Now let $\tau_{s}$ and $\tau_{p}$ denote the execution times for a base task in the serial and parallel stages, respectively. In the parallel processing stage, the total number of base tasks is $N_{p}=m_{p} \times n_{p}$, called the problem size, where $m_{p}$ is the number of parallel processing units (also called executors) or degree of parallelism (DOP); and $n_{p}$ is the

\footnotetext{
${ }^{1}$ The prediction errors at this level is considered small for resource provisioning [6].
}

number of base tasks mapped to and processed sequentially by each executor in the parallel stage, referred to as per-node workload level hereafter.

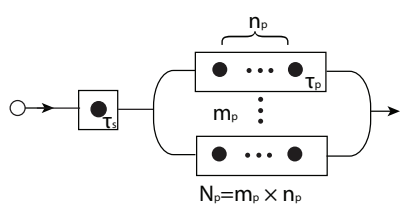

Figure 1: System Model for Amdahl's and Gustafson's laws. Each black dot represents a base task and each white box represents an executor. It has two stages, i.e., a serial and a parallel execution stage.

Now, the speedup, $S\left(N_{p}, m_{p}\right)$, for the above system model can be written as the following,

$$
S\left(N_{p}, m_{p}\right)=\frac{\tau_{s}+N_{p} \tau_{p}}{\tau_{s}+n_{p} \tau_{p}}
$$

where the numerator is the job response time when all the tasks are executed sequentially using a single executor and the denominator is the job execution time based on the above two-stage execution model. By writing down this formula, we implicitly assume that $\tau_{s}$ and $\tau_{p}$ stay the same for both sequential execution model and parallel execution model, which may not hold true in general, as we shall see. Now, both Amdahl's and Gustafson's laws can be easily derived from this expression, by taking into account of their respective workload models.

For fixed-sized workload, the problem size, $N_{p}$ is a constant, we have the following discretized Amdahl's law,

$$
S=\frac{1}{\frac{\eta}{m_{p}}+(1-\eta)}
$$

where,

$$
\eta=\frac{N_{p} \tau_{p}}{N_{p} \tau_{p}+\tau_{s}}
$$

is a constant. It simply states that for the fixed-size workload, the speedup is upper bounded by $\frac{1}{1-\eta}$, regardless of how many executors, $m_{p}$, are in use, where $m_{p} \leq N_{p}$. This is a discretized version of the original Amdahl's law. While the underlying parallelizable portion of the workload in the original Amdahl's law is infinitely divisible, the current one can only be divided into up to $N_{p}$ base tasks, which are processed by up to $m_{p}=N_{p}$ parallel executors.

For fixed-time workload, i.e., $n_{p}$ is a constant, we arrive at the following Gustafson's law,

$$
S=m_{p} \epsilon+(1-\epsilon)
$$

where,

$$
\epsilon=\frac{n_{p} \tau_{p}}{n_{p} \tau_{p}+\tau_{s}}
$$

is a constant. It simply states that for the fixed-time workload, the speedup grows unbounded, as both $m_{p}$ and $N_{p}$, increase at fixed $n_{p}=\frac{N_{p}}{m_{p}}$. Again, it is a discretized version of the original Gustafson's law, as the parallelizable portion of the workload 
mapped to any given executor, is measured in terms of an integer number of base tasks, i.e., the per-node workload level $n_{p}$. Traditionally, the two scaling laws are considered as two mutually exclusive views of scaling for parallel computing. Now, we identify the limitations of the system and workload models underlying the above scaling laws.

First, we observe that in the context of big data analytics workloads, the fixed-size and fixed-time workload models represent only two extreme scenarios of the same problem, i.e., resource-abundant and resource-constrained scenarios, respectively. By resource-abundant, we mean that the parallelizable portion of the workload can be processed in its entirety by just one executor, i.e., $m_{p}=1$ and $n_{p}=N_{p}$. In this case, one is interested in characterizing the scaling properties when the fraction of the parallelizable workload on each executor, $n_{p}=\frac{N_{p}}{m_{p}}$, decreases as the number of executors, $m_{p}$, increases at fixed $N_{p}$, leading to Amdahl's law in Eq. (2). By resource-constrained, we mean that each executor can only handle a fraction of the total parallelizable portion of the workload, $n_{p}=\frac{N_{p}}{m_{p}}$, e.g., the case when the memory allocated to each executor is fully occupied by the data block assigned to it. In this scenario, the workload grows with the number of allocated executors, $m_{p}$, resulting in Gustafson's law (Eq. (4)). Clearly, both scenarios may occur in practice, especially in a consolidated datacenter environment, where the resource availability may vary significantly from peak hours to off peak hours and the workload size may change dramatically from one application to another. Moreover, in general, as the system scales out, a big data analytics workload may scale in terms of both $N_{p}$ and $m_{p}$, not following any of these two workload models. Hence, from the job resource provisioning point of view, one would be interested in finding the maximum speedup trajectory as a function of $N_{p}$ and $m_{p}$. Clearly, the system model with workload discretization given in Figure 1 allows all possible trajectories to be characterized, not limited to the fixed-size or fixed-time workloads and hence, is a good model to allow a large workload design space to be exploited.

Second, we note that the base tasks for both parallel and serial portions of the workload underlying both Amdahl's and Gustafson's laws are fixed, i.e., $\tau_{p}$ and $\tau_{s}$ are constants and does not scale with $m_{p}$ or $N_{p}$. For big data analytics workloads, however, this is not the case in general. As we shall see in Section 4, both may scale with $N_{p}$ and/or $m_{p}$ for Spark-based multi-stage workloads.

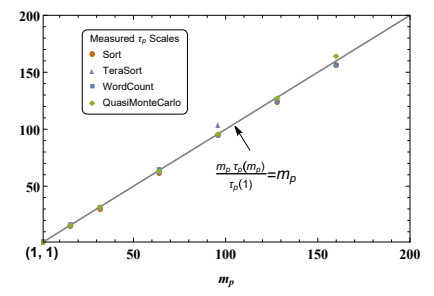

(a) Parallel Scaling

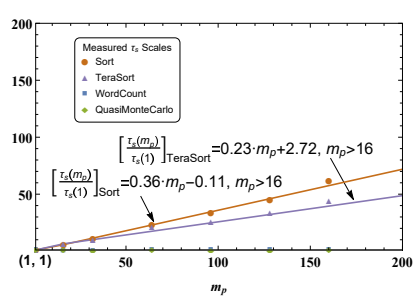

(b) Sequential Scaling
Figure 2: The scaling of serial and parallel portion of MapReduce workloads.
Even for some simple MapReduce-based applications, strong scalings for the serial portions of the workloads are found. For example, we do the experiments on Amazon EC2 cloud with EMR (elastic MapReduce) for four representative micro benchmarks from HiBench suite [7], including WordCount, Sort, TeraSort, and Quasi Monte Carlo (QMC) Pi program. We purposely configure only a single reducer in the Reduce phase so that the initialization and reducer phases together can mimic the serial stage in Figure 1. We consider the fixed-time workload by increasing the number of executors, $m_{p}$, in the Map phase with a $128 \mathrm{MB}$ data block for every executor added. Figure 2(a) and (b) depict the scaling of $r_{p}\left(m_{p}\right)=\frac{\tau_{p}\left(m_{p}\right)}{\tau_{p}(1)}$ and $r_{s}\left(m_{p}\right)=\frac{\tau_{s}\left(m_{p}\right)}{\tau_{s}(1)}$, assuming that $\tau_{p}=\tau_{p}\left(m_{p}\right)$ and $\tau_{s}=\tau_{s}\left(m_{p}\right)$. As one can see, while none of the four exhibit visible parallel scaling, both Sort and TeraSort see strong linear serial scalings with $m_{p}$. As a result, while WordCount and QMC Pi follow Gustafson's law and grow unbounded, Sort and TeraSort are upper bounded, as shown in Figure 3.

The above examples clearly indicate that a good scaling model for big data analytics workloads should take the possible scalings for both parallel and serial portion of the workload into account.

Third, as explained in the introduction section, big data analytics may involve many parallel execution stages in addition to the initialization stage. Also, as the problem size increases, the number of parallel execution stages may grow. Clearly, the system model in Figure 1 fails to capture these features.

The above fallacies in applying the traditional scaling laws to big data analytics workloads motivate the work presented in this paper.
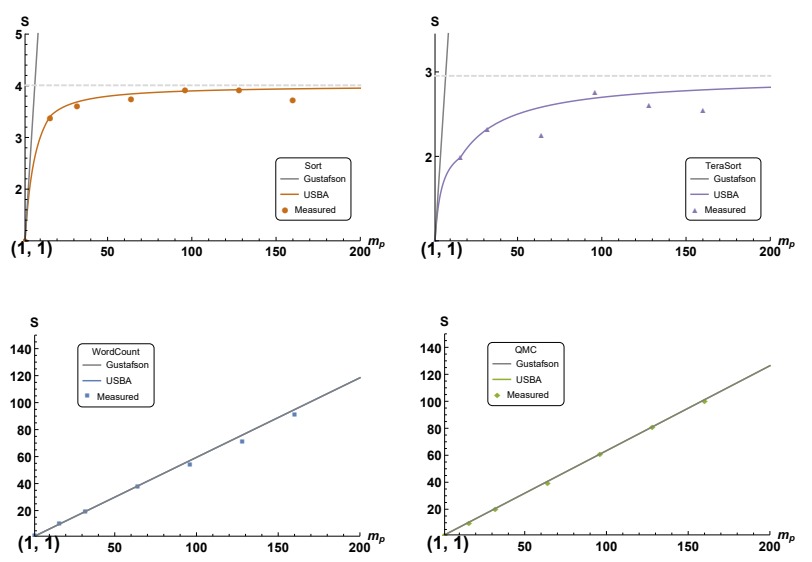

Figure 3: The speedups for four MapReduce benchmarks with fixed-time workloads. As shown, when taking serial scaling effects into account, big data analytics workloads may no longer follow Gustafson's law, i.e., the Sort and TeraSort cases.

\section{USBA}

In this section, we introduce the system and workload models, the speedup, and the regression model, separately.

\subsection{System Model with Discretized Workload}

Consider a system model with discretized workload, depicted in Figure 4. It generalizes the two-stage system model in Figure 1 to a $(L+1)$-stage system model with a serial initialization stage and 
$L$ parallel task execution stages with (the dotted vertical lines) or without synchronization barriers. At any given stage, $k$, there are $m_{k}$ executors working in parallel, each executing $n_{k}=\frac{N_{k}}{m_{k}}$ base tasks sequentially, where $N_{k}$ is the total workload or total number of base tasks at stage $k$.

Now, define $t_{k}\left(N_{k}, m_{k} ; i, j\right)$ to be the task execution time for the base task at position $j$ in the $i$ th executor at stage $k$, which in general is a random variable. As our experiment shows, it can be a strong function of the workload size, $N_{k}$, as well as parallel degree, $m_{k}$, at stage $k$. To limit the exposure, we further assume that all the base tasks at any given stage $k$ have the same mean task execution time, i.e., $\mathbb{E}\left[t_{k}\left(N_{k}, m_{k} ; i, j\right)\right]=\mathbb{E}\left[t_{k}\left(N_{k}, m_{k}\right)\right]$, for $i=1, \ldots, m_{k}$ and $j=1, \ldots, n_{k}$.

First, we note that in the above model, the serial initialization stage $k=0$ is modeled separately from the parallel execution stages, even though it could be treated as special case of a parallel execution stage with a single base task. As we shall see later, this is because the initialization stage exhibits totally different scaling properties from the parallel execution stages and hence, needs to be modeled and predicted separately from the rest of the stages.

Second, we note that the above model inherits the nice features from the model in Figure 1. In particular, it can accommodate various workload models, including the fixed-size workload (i.e., increasing $m_{k}$ at fixed $N_{k}$ for $\left.k=1, \ldots, L\right)$, fixed-time workload (i.e., increasing $N_{k}$ at fixed $n_{k}=\frac{N_{k}}{m_{k}}$ for $k=1, \ldots, L$ ), or any combinations of the two in a $2 L$-multidimensional space defined by $N_{1}, m_{1}, \ldots, N_{L}, m_{L}$. Moreover, it degenerates to the model in Figure 1 at $L=1$, meaning that Amdahl's and Gustafson's laws can be derived from the above model as special cases.

Finally, we note that the $2 L$ multidimensional space of the above model can be reduced to a two dimensional space, greatly simplifying the scaling analysis of the model. As we shall see later, for Spark-based workload, the workload size in terms of $N_{k}$ and $m_{k}$ at parallel execution stage $k$, for $k=1, \ldots, L$, are strongly correlated with the problem size, $N_{p}$, defined as the initial number of parallel

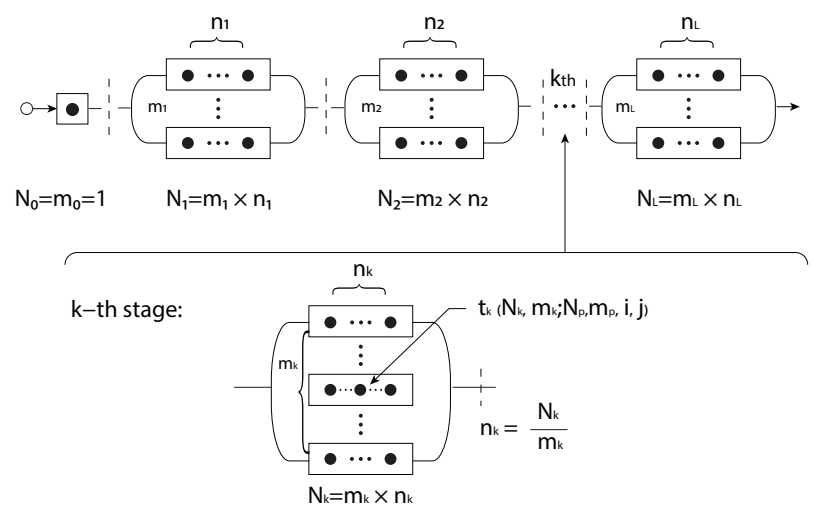

Figure 4: A system model with discretized workload. Stages are divided by data transformations that potentially involve large sequential overhead caused by the barrier, normally known as "shuffle" boundaries. Each white circle represents a driver process in that stage which takes input and dispatches tasks to its workers. The workers are usually running VM instances on core/task nodes of the cluster, depicted as white squire boxes in the figure. And each black circle represents a task being assigned to the worker. Also, when a multi-stage workload contains multiple chains of stages, the longest chain of stages with dependencies shall drive its performance in terms of execution latency. base tasks that a job spawns, as well as the degree of parallelism, $m_{p}$, i.e., the number of executors configured, where in general, $N_{1}=N_{p}$ and $m_{1}=m_{p}$. Moreover, the number of stages, $L$, may also be a function of $N_{p}$ and $m_{p}$. As we shall see, this observation lends us an effective means to characterize and predict the scaling properties of a big data analytics workload, using only two parameters, $N_{p}$ and $m_{p}$.

\subsection{Speedup}

The speedup for a given problem size must be measured against a baseline solution. Ideally, a baseline solution should follow a strictly sequential execution model, e.g., the sequential execution model in the case of Amdahl's or Gustafson's law. Unfortunately, for big data analytics, a strictly sequential execution model may not be implementable, as the most probable reason for scale-out processing of a big data workload is that both time and space complexities to do so sequentially are too high.

An alternative solution, e.g., proposed in [8], is to replace the baseline sequential model with a baseline scale-out model at the lowest possible DOP (degree of parallelism) that is implementable. For example, in the context of our USBA model, the speedup, $S_{r}$, thus defined can be written as,

$$
S_{r}\left(L,\left\{N_{k}, m_{k}\right\}\right)=\frac{\mathbb{E}\left[t_{0}\left(N_{1}, m_{1}^{o}\right)\right]+\mathbb{E}\left[T\left(L,\left\{N_{k}, m_{k}^{o}\right\}\right)\right]}{\mathbb{E}\left[t_{0}\left(N_{1}, m_{1}\right)\right]+\mathbb{E}\left[T\left(L,\left\{N_{k}, m_{k}\right\}\right)\right]}
$$

where $t_{0}\left(N_{1}, m_{1}\right)$ is the task response time in the initialization stage, which is a function of the problem size; $T\left(L,\left\{N_{k}, m_{k}\right\}\right)$ is the job response time with respect to the parallel execution stages; the numerator is the average job response time for the baseline scale-out configuration at the lowest DOP in terms of $m_{k}^{o}$ 's and the denominator is the average job response time for the desired scale-out configuration, where $m_{k}>m_{k}^{o}$ for $k=1, \ldots, L$.

Although offering a measurable speedup for a scale-out configuration versus a baseline one, the above definition suffers from a major drawback. As the problem size increases, the DOP for the baseline, i.e., $m_{k}^{o}$, s, must also increase to be implementable. Hence, the speedup becomes a relative measure depending on the range of problem sizes. This makes it difficult to identify the true performance bounds as problem size becomes large, as there is no fixed point of reference.

To address the above issue, USBA adopts the following ideal, easily implementable sequential execution model as the baseline. This baseline model simply uses the mean job response time measured at the minimum problem size, i.e., $N_{p}=1$ and $m_{p}=1$ (i.e., without scale-out effects), multiplied by $N_{p}$ as the mean job response time, $\mathbb{E}\left[T_{o}\left(N_{p}\right)\right]$, at any given problem size in terms of $N_{p}$, independent of $m_{p}$. More specifically, assume that $L=L\left(N_{p}, m_{p}\right)$ in general. At the smallest problem size, i.e., $N_{p}=1, m_{p}=1$ and $L=L(1,1)$, define $\tau_{k}$ to be the average task execution times, i.e., $\tau_{0}=\mathbb{E}\left[t_{0}(1,1)\right]$ and $\tau_{k}=\mathbb{E}\left[t_{k}(1,1)\right]$, for $k=1, \ldots, L(1,1)$. Then, we have,

$$
\mathbb{E}\left[T_{o}\left(N_{p}\right)\right]=N_{p} \times T(L(1,1),\{1,1\})=N_{p} \sum_{k=1}^{L(1,1)} \tau_{k}
$$


Clearly, this ideal sequential model is free from the scale-out effects, i.e., independent of $m_{p}$, and hence, is truly a pure sequential one.

With the above baseline sequential execution model, now we can define speedup, $S\left(L,\left\{N_{k}, m_{k}\right\}\right)$, as follows,

$$
S\left(L,\left\{N_{k}, m_{k}\right\}\right)=\frac{\mathbb{E}\left[t_{0}(1,1)\right]+\mathbb{E}\left[T_{o}\left(N_{p}\right)\right]}{\mathbb{E}\left[t_{0}\left(N_{1}, m_{1}\right)\right]+\mathbb{E}\left[T\left(L,\left\{N_{k}, m_{k}\right\}\right)\right]}
$$

Now, with reference to the model in Figure $4, \mathbb{E}\left[T\left(L,\left\{N_{k}, m_{k}\right\}\right)\right]$ can be written as follows,

$$
\mathbb{E}\left[T\left(L,\left\{N_{k}, m_{k}\right\}\right)\right]=\sum_{k=1}^{L} \mathbb{E}\left[R\left(k, N_{k}, m_{k}\right)\right]
$$

where $R\left(k, N_{k}, m_{k}\right)$ is the job response time with respect to stage $k$ and is given by,

$R\left(k, N_{k}, m_{k}\right)=\left\{\begin{array}{l}\max _{j \in\left[1, m_{k}\right]}\left\{\sum_{i=1}^{N_{k} / m_{k}} t_{k}\left(N_{k}, m_{k} ; i, j\right)\right\}, \\ \text { with barrier, } \\ \sum_{i=1}^{N_{k} / m_{k}} t_{k}\left(N_{k}, m_{k} ; i, 1\right), \\ \text { without barrier. }\end{array}\right.$

This expression accounts for the case with or without synchronization barrier. For the case with barrier, $R\left(k, N_{k}, m_{k}\right)$ is determined by the slowest task at stage $k$. This definition allows long-tail task execution effects, e.g., due to stragglers [9], to be captured. For the case without barrier, since the total mean response times for the $n_{k}$ base tasks at different parallel executors are the same, without loss of generality, $R\left(k, N_{k}, m_{k}\right)$ is expressed as the response time with respect to the first executor.

Clearly, with the above definition, the speedups for all the execution configurations in terms of $m_{k}$ 's can be compared, as they are measured against the same baseline. Moreover, there is an added benefit of this definition. Namely, the speedup in Eq. 6 can be derived from the current speedup as follows,

$$
S_{r}\left(L,\left\{N_{k}, m_{k}\right\}\right)=\frac{S\left(L,\left\{N_{k}, m_{k}\right\}\right)}{S\left(L,\left\{N_{k}, m_{k}^{o}\right\}\right)}
$$

Finally, we note that USBA is a unified scaling model that renders Amdahl's and Gustafson's scaling models its special cases, i.e., a deterministic version of USBA with $L=1$ and fixed-size workload for the Amdahl's case and fixed-time workload for the Gustafson's case.

\subsection{Regression Model}

In this paper, we focus on Spark-based big data analytics workloads. Spark is well known for its "In-Memory Computing" and outperform traditional MapReduce framework (e.g. Hadoop MapReduce) in processing large amount of data. As a result, it has been widely adopted in business applications in recent years. A Spark-based workload may contain up to thousands of processing stages, constructed by various parallel or sequential operations on RDDs (i.e., Resilient Distributed Datasets [10]) and the stages are bounded by data shuffle dependencies. These shuffle boundaries introduce barriers where a stage must wait for its previous (parent) stage to finish before it fetches the intermediate results. Each stage is associated with a certain number of tasks, which are assigned to a number of executors (an executor is a process on a worker node for computation and data storage) by the driver program. Therefore, Spark applications are by design multi-stage workloads that well match with the USBA scaling model.

In what follows, we use a machine learning benchmark from Intel Hibench Suite, i.e., Bayes Classifier (Bayes), to guide the discussion. The workload is a simple multi-class classification algorithm implemented in spark.mllib and uses automatically generated documents provided by the Hibench Suite as data input.

Figure 5 shows a workflow of Bayes as a serial executions of stages, which is typical for most Spark applications. In this example, $N_{p}=16, m_{p}=8$ and $L=13$ with a stage ID assigned to each stage, as shown in the leftmost column. We first note that the number of Succeeded/Total tasks in column three (i.e., $N_{k}$ ) at each stage is closely related to either $N_{p}$ or $m_{p}$. More specifically, it takes value of $N_{p}=16$ (in these stages $\left.m_{k}=8\right), m_{p}=8\left(m_{k}=8\right),\left(m_{p}-1\right)=7$ $\left(m_{k}=7\right)$, or $1\left(m_{k}=1\right)$, depending on the actual operators taken at that stage, as listed in the Description column. Our experiments also indicate that $L$ changes with $N_{p}$ and $m_{p}$ as well. The existence of $N_{k}, m_{k}$, and $L$ dependencies on $N_{p}$ and $m_{p}$ is the fundamental premise underlying the following regression models.

We define the following two scaling factors,

$$
\begin{aligned}
p\left(N_{p}, m_{p}\right) & =\frac{\mathbb{E}\left[T\left(L,\left\{N_{k}, m_{k}\right\}\right)\right]}{\sum_{k=1}^{L(1,1)} \tau_{k}} \\
q\left(N_{p}, m_{p}\right) & =\frac{\mathbb{E}\left[t_{0}\left(N_{p}, m_{p}\right)\right]}{\tau_{0}}
\end{aligned}
$$

called $p$-function and $q$-function, respectively. In general, $p\left(N_{p}, m_{p}\right) \geq$ 1 and $q\left(N_{p}, m_{p}\right) \geq 1$. Note that the above variable deduction is made possible because $L, N_{k}$ and $m_{k}$ (for $k=1, \ldots, L$ ) are implicit functions of $N_{p}$ and $m_{p}$. As a result, $S\left(L,\left\{N_{k}, m_{k}\right\}\right)=S\left(N_{p}, m_{p}\right)$ and Eq. (8) can be rewritten as follows,

$$
S\left(N_{p}, m_{p}\right)=\frac{\tau_{0}+N_{p} \sum_{k=1}^{L(1,1)} \tau_{k}}{\tau_{0} \times q\left(N_{p}, m_{p}\right)+\left(\sum_{k=1}^{L(1,1)} \tau_{k}\right) \times p\left(N_{p}, m_{p}\right)}
$$

since $\tau_{k}$ (for $k=0,1, \ldots, L(1,1)$ ) are easily measurable parameters at the smallest problem size $N_{p}=1$ without scaling out (i.e., $\left.m_{p}=1\right)$, the speedup $S\left(N_{p}, m_{p}\right)$ is completely determined by the $p$-function and $q$-function. In other words, if one can pin down these functions, e.g., by regression, based on measured data at small $N_{p}$ 's and $m_{p}$ 's, the scaling behaviors in terms of speedup can then be predicted at larger $N_{p}$ and $m_{p}$. To this end, we propose the following solution.

Without using any structural information from the workflow, we identify the most plausible function format for $p$-function by searching in a large function space using the $3 \mathrm{D}\left(\left\{S\left(N_{p}, m_{p}\right), N_{p}, m_{p}\right\}\right)$ measured data as input. This approach, if successful, can be generally applied to any applications and programming frameworks that match the USBA scaling model, not limited to Spark-based data mining and graph applications under study. To this end, we resort to an open source online curve (and surface) fitting tool [11]. Using our 3D data set as input, it compares thousands of templates selected from a 


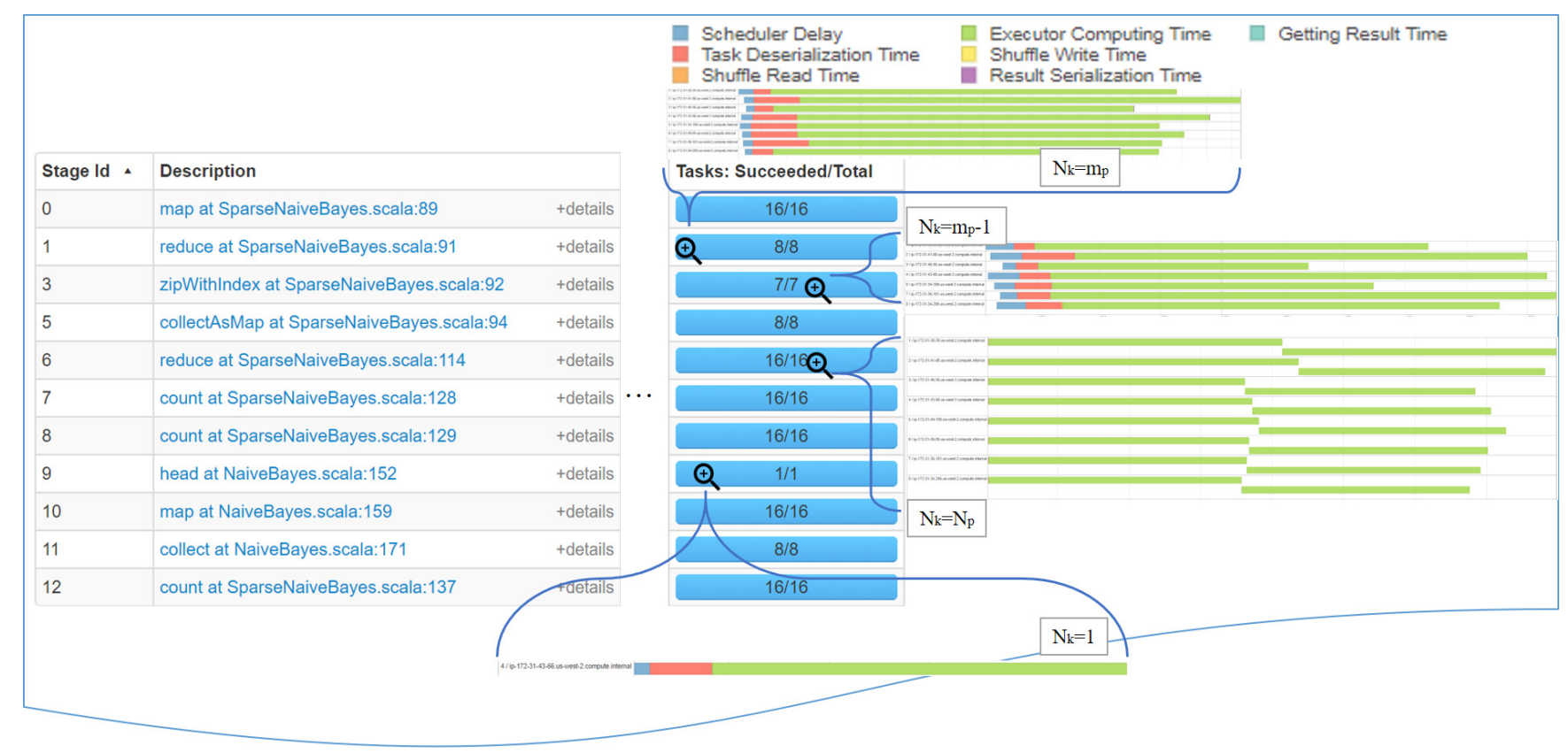

Figure 5: Workflow in stages for Bayes. The number of tasks $\left(N_{k}\right)$ and executors $\left(m_{k}\right)$ within each stage are closely related to $N_{p}$ and $m_{p}$.

wide range of families (including Polynomial, Logarithmic, Exponential, Miscellaneous, Power, etc.) in parallel. Taking the results from the top of the list, we then examine the relative magnitudes of the involved parameters to further trim the function to a meaningful and simple form:

$$
p\left(N_{p}, m_{p}\right)=(1-\beta-\gamma)+\alpha \cdot \log \left(\frac{N_{p}}{m_{p}}\right)+\beta \cdot m_{p}+\gamma \cdot N_{p}
$$

Although Eq. (15) is derived without taking the underlying workflow structure into account, it is still plausible to interpret the meanings for each involved terms in this expression. The term, $(\alpha$. $\left.\log \left(\frac{N_{p}}{m_{p}}\right)\right)$, indicates that the effective computing time for the workload mapped to each executor increases fast at small load level, $n_{p}$, but then increases sublinearly fast as $n_{p}$ becomes large. This can be understood by the fact that the initial base task execution incurs much more overhead than the following base tasks as is evident in the example given in Figure 8. The term, $\beta \cdot m_{p}$, is clearly the overheads induced by the scaling out, e.g., the communication overheads. The term, $\gamma \cdot N_{p}$, which is a very small negative term, is probably an artifact induced by the pseudo random nature of the synthetic data set generated by the HiBench tool itself [7].

The above expression bears some resemblance to the response time model derived from the workflow structures underlying some Spark-based applications (Equation 1 in [6]). A major difference lies in the term for per executor workload computing time. In [6], this term is given as a linear function of $n_{k}$, whereas ours is a sublinear one in log form. Comparison study of the two solutions with application to the four Spark benchmarks (not shown here) indicates that our solution outperforms the other one by a small margin. This suggests that a workflow-structure-oblivious solution like ours can perform equally well as a more elaborate workflowstructure-aware solution.

With regard to $q$-function, we find from the raw data for all four Spark benchmarks that it is only a function of $m_{p}$, not $N_{p}$ and it is a strong piecewise function, which can be easily modeled using the following expression,

$$
q\left(m_{p}\right)= \begin{cases}1-\epsilon+\epsilon \cdot m_{p} & m_{p} \leq \theta \\ 1-\epsilon+\epsilon \cdot \theta & \text { o.w. }\end{cases}
$$

It means that the launch overhead increases with $m_{p}$ initially and then reach a threshold $\theta$, beyond which it becomes a constant. Note that in [6], this term is assumed to be a constant.

In Table 1, we list the parameter values for all the parameters in $p$ function and q-function, together with their respective significances, i.e., the $p$-values, for both performance modeling and prediction, estimated by Wolfram Mathematica [12]. Generally speaking, $p$ value $\leq 0.05$ implies the estimated value is significant. The $p$-values for all the parameters except $\gamma$ 's for some benchmarks indicate that they are significant and cannot be omitted. The estimated small $\gamma$ values and their relatively large $p$-values also suggestions that the impact of the artifacts induced by the pseudo randomness of the synthetic data is minor.

Finally, we need to point out that there are other ways of generating the regression models that we tested. For instance, RSM (Response Surface Methodology) [13] is a widely adopted 3D curve fitting technique which is particularly useful in searching for optimal values. Unfortunately, even though the model is systematic and easy to follow, it fails at prediction with large percentage errors in our use cases. 
Table 1: Parameter estimation for the selected Spark benchmarks' $p$-function and $q$-function.

(a) Performance Modeling

\begin{tabular}{|c|c|c|c|c|c|c|c|c|c|c|}
\hline & \multicolumn{2}{|r|}{$\alpha$} & \multicolumn{2}{|c|}{$\beta$} & \multicolumn{2}{|c|}{$\gamma$} & \multicolumn{2}{|c|}{$\epsilon$} & \multicolumn{2}{|c|}{$\theta$} \\
\hline & est. & p-value & est. & p-value & est. & p-value & est. & p-value & est. & p-value \\
\hline Bayes & 1.58 & $3.15 \mathrm{E}-11$ & 0.051 & $4.90 \mathrm{E}-19$ & -0.006 & 0.008 & 0.044 & $1.58 \mathrm{E}-27$ & 19.09 & $1.84 \mathrm{E}-27$ \\
\hline $\mathrm{RF}$ & 1.938 & $1.19 \mathrm{E}-13$ & 0.074 & $2.89 \mathrm{E}-24$ & -0.009 & 0.000116 & 0.04389 & $2.29 \mathrm{E}-34$ & 18.983 & $2.71 \mathrm{E}-34$ \\
\hline SVM & 0.807 & 0.006402 & 0.14 & $1.28 \mathrm{E}-33$ & - & - & 0.037 & $1.50 \mathrm{E}-32$ & 19.905 & $1.62 \mathrm{E}-32$ \\
\hline NWeight & 2.505 & 8.39E-09 & 0.106 & $6.82 \mathrm{E}-20$ & -0.022 & $1.05 \mathrm{E}-05$ & 0.04 & $1.82 \mathrm{E}-29$ & 19.516 & $1.83 \mathrm{E}-29$ \\
\hline
\end{tabular}

(b) Prediction $\left(N_{p}=64\right)$

\begin{tabular}{|c|c|c|c|c|c|c|c|c|c|c|}
\hline & \multicolumn{2}{|r|}{$\alpha$} & \multicolumn{2}{|r|}{$\beta$} & \multicolumn{2}{|c|}{$\gamma$} & \multicolumn{2}{|r|}{$\epsilon$} & \multicolumn{2}{|c|}{$\theta$} \\
\hline & est. & p-value & est. & p-value & est. & $p$-value & est. & $\mathrm{p}$-value & est. & p-value \\
\hline Bayes & 1.555 & $4.22 \mathrm{E}-11$ & 0.031 & 0.000443 & $-1.91 \mathrm{E}-06$ & 0.999713 & 0.050 & $2.41 \mathrm{E}-08$ & 15.367 & $2.40 \mathrm{E}-08$ \\
\hline $\mathrm{RF}$ & 2.194 & 6.61E-09 & 0.085 & $1.77 \mathrm{E}-05$ & -0.021 & 0.067175 & 0.049 & $7.08 \mathrm{E}-10$ & 15.612 & $6.93 \mathrm{E}-10$ \\
\hline SVM & 0.886 & $1.47 \mathrm{E}-05$ & 0.137 & $2.08 \mathrm{E}-13$ & - & - & 0.037 & $4.54 \mathrm{E}-16$ & 19.783 & $2.08 \mathrm{E}-14$ \\
\hline NWeight & 2.067 & 1.09E-05 & 0.09 & 0.00136 & $-1.09 \mathrm{E}-07$ & 0.999995 & 0.040 & $1.81 \mathrm{E}-14$ & 19.044 & $6.69 \mathrm{E}-13$ \\
\hline
\end{tabular}

Table 2: Measurements of terms in numerator and denominator of Eq. (14) from Spark Log files.

\begin{tabular}{|l|l|l|}
\hline Term & Log File Identifier & Configuration \\
\hline$\tau_{0}$ & stage_start_time - app_launch_time $($ first stage $)$ & $N_{p}=m_{p}=1$ \\
\hline$\tau_{k}(k \geq 1)$ & stage_end_time - stage_start_time & $N_{p}=m_{p}=1$ \\
\hline$q\left(m_{p}\right) \times \tau_{0}$ & stage_start_time - app_launch_time $($ first stage $)$ & $N_{p} \geq m_{p}>1$ \\
\hline$p\left(N_{p}, m_{p}\right) \times\left(\sum_{k=1}^{L(1,1)} \tau_{k}\right)$ & Accumulate $\{$ stage_end_time - stage_start_time\} & $N_{p} \geq m_{p}>1$ \\
\hline
\end{tabular}

\section{APPLICATION OF USBA}

This section applies USBA to four selected Spark benchmarks from Intel HiBench Suite. We explain the experiment setup on the EC2 cloud; the measurement procedure; and the results separately.

\subsection{Experiment Setup}

We deployed the Intel HiBench suite on EC2 with the EMR (Elastic MapReduce) service. The selected benchmarks includes three machine learning applications:Bayes Classifier (Bayes), Random Forest $(R F)$, Support Vector Machine(SVM), and a graph application (NWeight). On the hardware side, a heterogeneous cluster, composed of one master node of m4.2xlarge instance type (8 vCPU, 32GB memory) and multiple core nodes of m4.large instance type (2 vCPU, 8GB memory), is set up. All the instances are pre-configured with sufficient storage capacity (100GB per-node) and bandwidth ( $\geq 450 \mathrm{Mbps}$ ). On the software side, dynamic resource allocation (i.e., performance tuning option) is turned off to prevent possible unpredictable execution behaviors, as executor reuse and logging is enabled for performance metrics collection. All the input data sets are generated by the respective data generators provided by this benchmark suite. The number of executors $\left(m_{p}\right)$ are chosen as $1,1 / 2$, $1 / 4$ and $1 / 8$ in ratio to $N_{p}$, or equivalently, $n_{k}=1,2,4$ and 8 .

\subsection{Measurement}

The next step is to extract the execution latencies for all stages from the application's Log file to derive the speedup in Eq. 14. Table 2 summarizes the terms in Eq. 14 that need to be measured. This done by tracing the beginning and ending timestamps for each execution stage in the Spark Log files, which are available in the format of JSON entries. Figure 6 illustrates these highlighted JSON entries and the ways to extract each timestamp.

\subsection{Results and Analysis}

Using the regression model introduced in Section 4.2, each benchmark's speedup can be explicitly evaluated as a function of the problem size $\left(N_{p}\right)$ and parallel degree $\left(m_{p}\right)$. Partial results are given in Table 3. As one can see, the the regression-model-based speedups are within $15 \%$ errors of the measured ones for the left three data mining benchmarks. For the graph benchmark on the right, they are within $21 \%$ errors. This suggests that the subtle differences of the underlying workflow structures for different types of applications may play an important role in modeling data analytics applications.

Now Figure 7 visualizes the complete results in the 3-D plots. As one can see, the model-based speedups closely follow the measured ones. Note that the contour in each plot has a peaked trajectory along which the speedups are maximized for the corresponding problem size $N_{p}$ and degree of parallelism $m_{p}$. From job resource

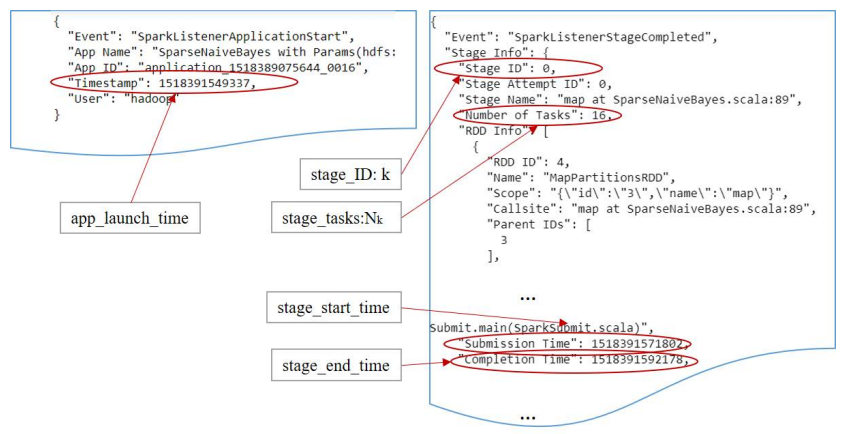

Figure 6: Log analyzer of Spark applications. As marked in the figure, for the $k_{t h}$ stage, it is tagged by the stage_tasks: $N_{k}$. The stage execution latency is calculated by stage_end_time stage_start_time, and the application launch overhead is estimated by the stage_start_time of the first launched Spark stage subtracted by app_launch_time. To distinguish the cost, we mark this phase of execution as the first stage and the rest regular stages afterwards. 
Table 3: Measured (partial) USBA speedups for Spark benchmarks in comparison with model estimations as problem size scaling from 1 to 256.

\begin{tabular}{|c|c|c|c|c|c|c|c|c|c|c|c|c|c|}
\hline \multirow[b]{2}{*}{$\mathrm{N}$} & \multirow[b]{2}{*}{$\mathrm{m}$} & \multicolumn{3}{|c|}{ Bayes } & \multicolumn{3}{|c|}{$\mathrm{RF}$} & \multicolumn{3}{|c|}{ SVM } & \multicolumn{3}{|c|}{ NWeight } \\
\hline & & exp. & USBA & $\mathrm{RE}$ & exp. & USBA & RE & exp. & USBA & $\mathrm{RE}$ & exp. & USBA & RE \\
\hline 1 & 1 & 1.0 & 1.0 & $0 \%$ & 1.0 & 1.0 & $0 \%$ & 1.0 & 1.0 & $2 \%$ & 1.0 & 1.0 & $1 \%$ \\
\hline 8 & 2 & 2.2 & 2.3 & $4 \%$ & 1.8 & 1.9 & $5 \%$ & 2.3 & 2.2 & $3 \%$ & 2.1 & 1.7 & $20 \%$ \\
\hline 8 & 4 & 3.3 & 2.9 & $12 \%$ & 2.2 & 2.5 & $14 \%$ & 2.4 & 2.2 & $11 \%$ & 2.7 & 2.3 & $16 \%$ \\
\hline 8 & 8 & 4.2 & 4.0 & $4 \%$ & 2.9 & 3.3 & $14 \%$ & 2.2 & 2.0 & $7 \%$ & 3.3 & 3.3 & $2 \%$ \\
\hline 64 & 32 & 17.0 & 15.3 & $10 \%$ & 12.3 & 12.0 & $3 \%$ & 7.3 & 7.0 & $4 \%$ & 10.0 & 11.0 & $10 \%$ \\
\hline 64 & 64 & 15.8 & 13.4 & $15 \%$ & 10.0 & 9.9 & $1 \%$ & 4.6 & 4.9 & $5 \%$ & 8.7 & 8.5 & $3 \%$ \\
\hline 96 & 48 & 23.3 & 19.9 & $15 \%$ & 16.0 & 15.2 & $5 \%$ & 8.0 & 8.3 & $4 \%$ & 14.2 & 13.9 & $2 \%$ \\
\hline 96 & 96 & 18.1 & 15.4 & $15 \%$ & 11.4 & 11.2 & $2 \%$ & 5.3 & 5.4 & $3 \%$ & 8.6 & 9.3 & $9 \%$ \\
\hline 128 & 32 & 23.6 & 26.2 & $11 \%$ & 21.8 & 21.0 & $4 \%$ & 13.5 & 13.1 & $3 \%$ & 17.2 & 20.7 & $21 \%$ \\
\hline 128 & 64 & 25.1 & 23.4 & $7 \%$ & 18.7 & 17.7 & $5 \%$ & 8.8 & 9.2 & $5 \%$ & 18.3 & 16.1 & $12 \%$ \\
\hline 128 & 128 & 16.7 & 16.7 & $0 \%$ & 12.8 & 12.0 & $6 \%$ & 6.1 & 5.7 & $6 \%$ & 10.3 & 9.8 & $4 \%$ \\
\hline 192 & 48 & 34.0 & 35.9 & $6 \%$ & 28.9 & 28.4 & $2 \%$ & 16.7 & 15.8 & $5 \%$ & 27.2 & 29.3 & $8 \%$ \\
\hline 192 & 96 & 31.6 & 28.5 & $10 \%$ & 22.0 & 21.2 & $3 \%$ & 9.6 & 10.4 & $9 \%$ & 21.5 & 19.2 & $11 \%$ \\
\hline 192 & 192 & 18.4 & 18.2 & $1 \%$ & 13.5 & 13.0 & $4 \%$ & 7.2 & 6.1 & $15 \%$ & 10.5 & 10.4 & $1 \%$ \\
\hline 256 & 64 & 38.8 & 44.2 & $14 \%$ & 33.9 & 34.7 & $2 \%$ & 18.5 & 17.7 & $4 \%$ & 32.6 & 37.0 & $14 \%$ \\
\hline 256 & 128 & 32.1 & 32.0 & $0 \%$ & 21.6 & 23.6 & $9 \%$ & 10.4 & 11.1 & $7 \%$ & 22.7 & 21.3 & $6 \%$ \\
\hline 256 & 256 & 16.8 & 19.1 & $14 \%$ & 14.0 & 13.5 & $4 \%$ & 5.9 & 6.3 & $7 \%$ & 10.0 & 10.7 & $8 \%$ \\
\hline
\end{tabular}

provisioning point of view, a job resource provisioning algorithm can be developed to identify the optimal point on this trajectory, based on the resource availability and/or monetary budget, which however, is out of scope of this paper.

Viewing clearly from this solution space, we are particularly interested in the scaling properties of the workload projected onto $m_{p}$ axis and $n_{p}=\frac{N_{p}}{m_{p}}$ axis, corresponding to the fixed-time workload underlying Gustafson's law and fixed-size workload underlying Amdahl's law. For the first scenario (i.e. fixed-time), it's easily to identify from the figure that at given number of worker nodes or degree of parallelism (DOP), $m_{p}$, the higher the per-node workload level, $n_{p}=\frac{N_{p}}{m_{p}}$, the better the performance is. In other words, the speedup curve at $n_{p}=4$ is higher than that at $n_{p}=2$, which in turn, is higher than that at $n_{p}=1$. This is due to the increased percentage of scale-out workload as the number of base tasks per executor decreases. This is evident from Figure 8 , in which stage 8 with per-node workload level of $2\left(\frac{N_{p}}{m_{p}}=2\right)$ is given. It clearly shows that the scheduling and deserialization time (i.e., the communication cost) of the first round of tasks outweigh the second one. Hence, further increasing per-node workload level effectively reduces the scale-out induced workload per base task. On the other hand, for the latter scenario (i.e. fixed-size), one can find out that except for SVM, all the other applications have their speedups peaks and then fall as DOP increases. For SVM however, the speedup decreases monotonically (for $m_{p} \geq 3$ ). This, again, is in stark contrast with that predicted by Amdahl's law, i.e., the speedup monotonically increases with a fixed upper bound as given in Eq. (2). This is due to the scale-out induced overhead, as discussed above. Again, the optimal configuration should be one with the per-node workload level matching the node-level resource availability.

\subsection{Prediction}

While modeling is important to understand the big data application's scaling behavior, speedup prediction on the other hand is useful for the system designers to achieve desired performance while avoid

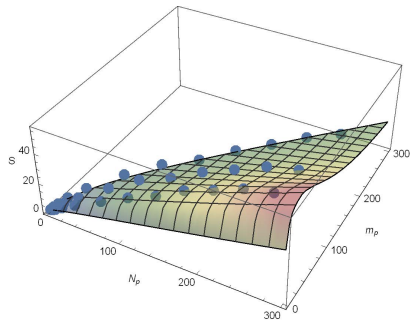

(a) Bayes

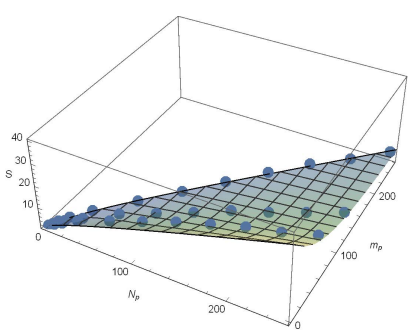

(c) SVM

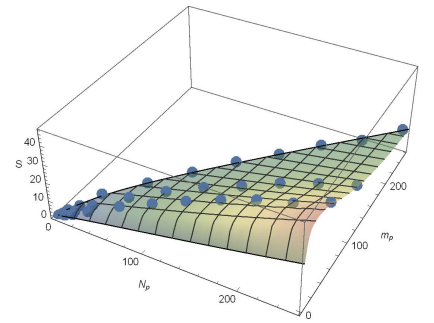

(b) RF

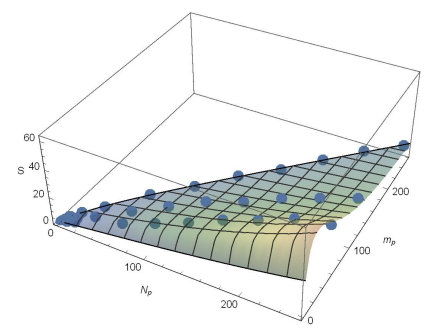

(d) NWeight
Figure 7: The 3D unified speedup solution spaces of all four Spark benchmarks. The highlighted surfaces represent the speedup solutions while the blue dots represents the experiment data collected (taken as the average values of multiple observations). The gradually-changed color planes from cold to warm indicate the "backbone" areas where the local performance peaks can be achieved.

wasting time and energy on over-allocating most valuable parallel computing resources.

Typically, using regression analysis or machine learning for prediction, a proper selection of the training data set is at the core of achieving the desired precision goal. In this study, we train our regression models introduced in Section 3.3 for the selected benchmarks at roughly $10 \%$ of total cost in terms of EC2 instance hours. The samples selected in the training set needs to cover all the scaling factors and hence, a certain threshold of the sample set size must 


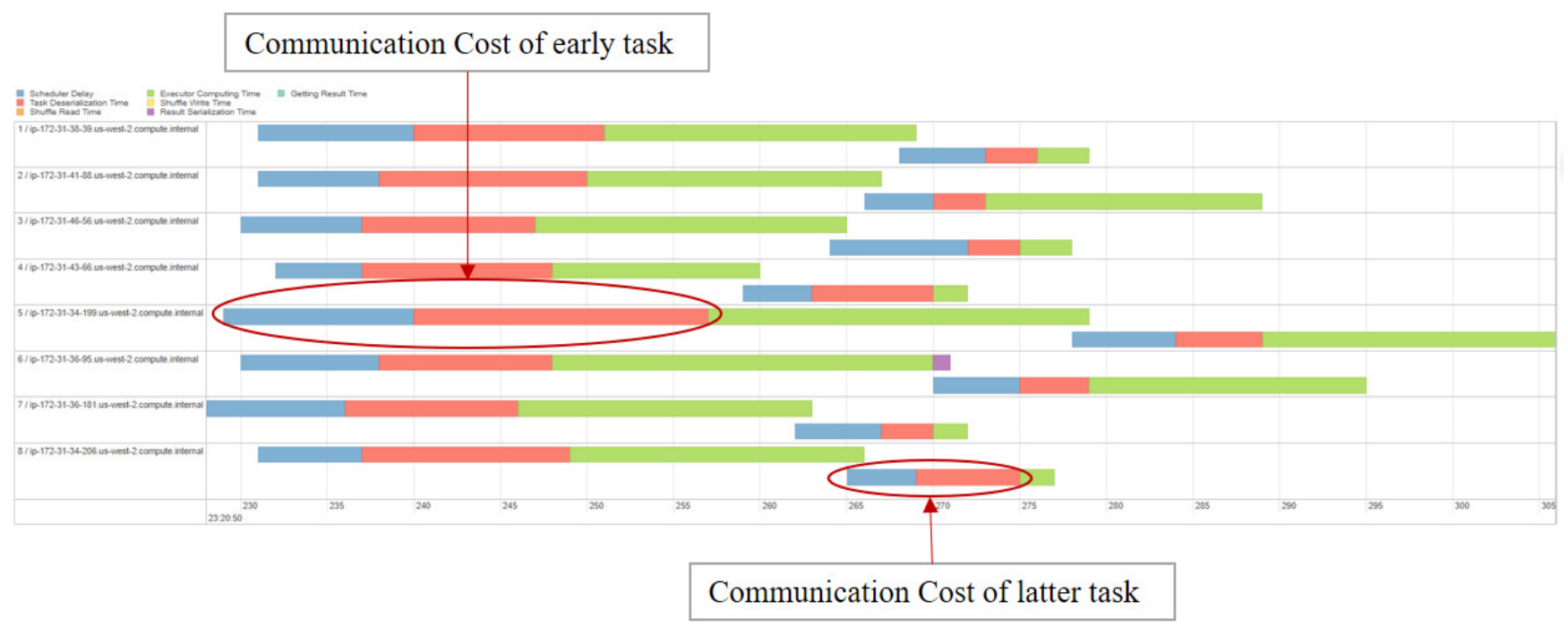

Figure 8: Communication and computing costs for a typical Spark application, the parallel degree is set as 2 for this workload.

be reached. Specifically, we use the data set for the problem size no bigger than $N_{p}=64$ as the training set. This is because we find that the fourth term in Eq. $(15)-\left(\gamma \cdot N_{p}\right)$ can only be captured when the problem size exceeds 32 , thus the data samples for $N_{p}=64$ have to be included in the training sets for Bayes, $R F$ and NWeight. But for $S V M$, the training set that covers only up to $N_{p}=16$ is sufficient for accurate prediction. This is because for $S V M$, the fourth term in p-function is insignificant, as is evident from Table 1 . This phenomenon can be possibly explained by the characteristics of synthetic input data sets. The contents of these pseudo-randomly generated data blocks inevitably repeat themselves (in cycles) as the problem size reaches certain level. This may cause the content-sensitive analytics programs to exhibit different scaling characteristics when the problem size reaches certain threshold, which cannot be predicted with the training samples from the problem size below this threshold.

Figure 9 demonstrates that except for a few outliers, most experimental data points are closely scattered around the prediction surface. In fact, the average prediction errors against the measured ones are constantly within $6 \%$ to $12 \%$ for the Spark machine learning benchmarks(Bayes, $R F$ and $S V M$ ) and $19 \%$ for the graph benchmark $-N W e i g h t$. The performance difference indicates the workload for NWeight may be more content sensitive than the rest machine learning benchmarks, and thus requires a wider training set coverage. Nevertheless, at this error range, the prediction model is sufficient to capture the speedup trending at large problem sizes. In fact, the performance of our prediction is on par with that of the workflowstructure-aware prediction model given in [6], which reports $20 \%$ prediction errors for big data analytics workloads.

In summary, USBA can be applied to the evaluation of the scaling properties for today's big data applications. It can also be used to facilitate the development of efficient job resource provisioning algorithms.

\section{RELATED WORK}

In this section, we first review the related work in terms of scaling modeling and then relevant job resource provisioning mechanisms.

As we have seen in the previous sections, big data analytics workloads cannot be adequately characterized by the existing scaling laws. However, to the best of our knowledge, to date, no scaling model is available that can adequately characterized such workloads. As a result, Amdahl's law is still the most widely cited law for big data analytics, e.g., [14],[8].

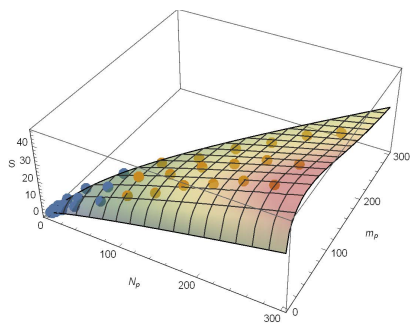

(a) Bayes

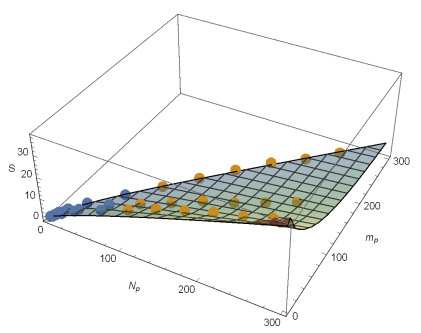

(c) SVM

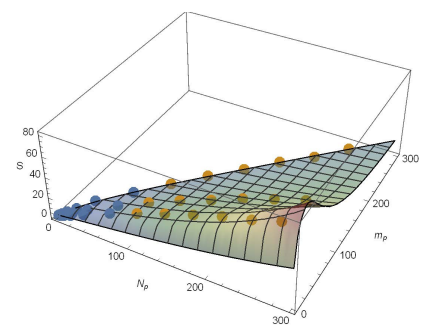

(b) $\mathrm{RF}$

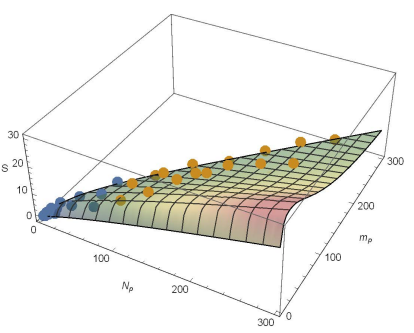

(d) NWeight
Figure 9: Speedup predictions for selected Spark benchmarks. The surface plots depicts the unified speedups calculated by prediction models derived from training samples $\left(N_{p} \leq 64\right)$, where these data collections are colored in blue. The rest samples (points colored in orange) are shown as the prediction targets. 
The lack of a scaling model does not mean that researchers are unaware of the inadequacy of the existing scaling models. In fact, as scale-out applications has emerged as the dominant applications in datacenter, rich scaling properties of such applications reveal themselves, which are well noted. Here are some examples. It was found [15] that for a fixed-size iterative computing and broadcast scale-out workload, the job stops scaling at about $n=60$, beyond which the speedup decreases due to linear increase of the broadcast overhead, where $n$ is the number of computing nodes for parallel processing. TCP-incast overhead was found to be responsible for the speedup reduction for many big data analytics applications [16]. Centralized job schedulers used in some popular programming frameworks, such as Hadoop and Spark, were found to pose performance bottlenecks for job scaling, due to a quadratic increase of the task scheduling rate as $n$ increases [17]. The scaling analysis of data mining applications [18] reveals that the reduction operations in each merging phase are induced by external scaling, resulting in much lower speedup than that predicted by Amdahl's law. As we demonstrated in Section 2, even for some simple MapReduce-based applications, including Sort and TeraSort (Hadoop), their scaling properties cannot be captured by the existing scaling laws.

Some results on the improvement of the existing scaling laws are available in the context of using multicore processors for parallel computing, e.g., considering multicore hardware scaling [19], multithreading and multicore scaling [20], and impact of critical section [20, 21]. However, the system and workload models underlying these scaling models still follow the ones for the traditional scaling laws, and hence, these scaling models are not suitable for the big data analytics workloads.

Job resource provisioning solutions can be generally classified into two categories, i.e., blackbox and graybox ones. The blackbox solutions generally rely on job profiling data (e.g., [22])as input to some machine learning or datamining algorithms to predict the resource demand of a target job to be scheduled, e.g. [23]. Although applicable to a wide range of applications, a blackbox solution generally works for recurring jobs only. The graybox solution makes use of the system and workload information to a certain degree so that some modeling can be done to narrow down the design space to allow lower cost of profiling/sampling for the prediction, e.g., [6], [24]. No white-box solution is available due to scale and complexity involved in a job workflow. Although without considering detailed resources, among other things, USBA by itself serve as a job resource provisioning solution, its system, workload, and prediction models have the potential to lead to an effective graybox job resource provisioning solution. In particular, the ability to capture the performance of different workload scalings from the resourceabundant, all the way to resource-constrained scenarios makes the job resource provisioning in the largest design space of practical interests possible.

\section{CONCLUSION}

In this paper, we proposed a new scaling model for scale-out datacenter applications, called Unified Scaling Model for Big data Analytics (USBA). USBA is a unified scaling model that applies to the modern big data analytics based on multi-stage iterative parallel programming frameworks and takes the traditional two-stage serial-and-parallel execution model as its special case. USBA also provides a regression model for the matching and prediction of the parallel speedup for big data analytics workloads. The case studies based on four Spark-based data mining and graph benchmarks demonstrated that USBA can capture the novel scaling properties of big data analytics workloads, which cannot be predicted by the traditional scaling laws, including Amdahl's and Gustafson's laws. These case studies also demonstrated the promising prospects of USBA to facilitate effective job resource provisioning for big data analytics workloads in datacenter environments.

\section{REFERENCES}

[1] Jeffrey Dean and Sanjay Ghemawat. MapReduce: Simplified Data Processing on Large Clusters. In Proceedings of the 6th Symposium on Operating Systems Design and Implementation - OSDI '04, pages 137-150, 2004.

[2] Matei Z. An Architecture for Fast and General Data Processing on Large Clusters. $\mathrm{PhD}$ thesis, University of California, Berkeley, 2013.

[3] Michael Isard, Mihai Budiu, Yuan Yu, Andrew Birrell, and Dennis Fetterly. Dryad: Distributed Data-parallel Programs from Sequential Building Blocks. In Proceedings of the 2nd ACM SIGOPS/EuroSys European Conference on Computer Systems 2007, EuroSys '07, pages 59-72, 2007.

[4] Gene M. Amdahl. Validity of the single processor approach to achieving large scale computing capabilities. In Proceedings of Am. Federation of Infomation Processing Societies Conf., pages 483-485. ACM, 1967.

[5] John L. Gustafson. Reevaluating Amdahl's law. Communications of the ACM, 31(5):532-533, 1988

[6] Shivaram Venkataraman, Zongheng Yang, Michael J Franklin, Benjamin Recht, and Ion Stoica. Ernest: Efficient performance prediction for large-scale advanced analytics. In NSDI, pages 363-378, 2016.

[7] Shengsheng Huang, Jie Huang, Jinquan Dai, Tao Xie, and Bo Huang. The hibench benchmark suite: Characterization of the mapreduce-based data analysis. In Data Engineering Workshops (ICDEW), 2010 IEEE 26th International Conference on, pages 41-51. IEEE, 2010.

[8] Isaac Triguero, Daniel Peralta, Jaume Bacardit, Salvador García, and Francisco Herrera. Mrpr: a mapreduce solution for prototype reduction in big data classification. neurocomputing, 150:331-345, 2015.

[9] Matei Zaharia, Andy Konwinski, Anthony D Joseph, Randy H Katz, and Ion Stoica. Improving mapreduce performance in heterogeneous environments. In Osdi, volume 8, page 7, 2008.

[10] Matei Zaharia, Mosharaf Chowdhury, Tathagata Das, Ankur Dave, Justin Ma, Murphy McCauley, Michael J Franklin, Scott Shenker, and Ion Stoica. Resilient distributed datasets: A fault-tolerant abstraction for in-memory cluster computing. In Proceedings of the 9th USENIX conference on Networked Systems Design and Implementation, pages 2-2. USENIX Association, 2012.

[11] James R Phillips. Zunzun. com online curve fitting and surface fitting web site. United States, 2012.

[12] Stephen Wolfram. The mathematica. Cambridge university press Cambridge, 1999.

[13] NR Draper. Response surface methodology: Process and product optimization using designed experiments: Rh myers and dc montgomery,(wiley, new york, 1995, isbn: 0471581003, pp. 714), 1997.

[14] Daniel Richins, Tahrina Ahmed, Russell Clapp, and Vijay Janapa Reddi. Amdahl's law in big data analytics: Alive and kicking in tpcx-bb (bigbench). In High Performance Computer Architecture (HPCA), 2018 IEEE International Symposium on, pages 630-642. IEEE, 2018.

[15] Mosharaf Chowdhury, Matei Zaharia, Justin Ma, Michael I. Jordan, and Ion Stoica. Managing Data Transfers in Computer Clusters with Orchestra. In Proceedings of the ACM SIGCOMM 2011 Conference, SIGCOMM '11, pages 98-109, 2011.

[16] Yanpei Chen, Rean Griffith, David Zats, Anthony D. Joseph, and Randy Katz. Understanding TCP incast and its implications for big data workloads. ¡login:, 37(3):24-38, 2012.

[17] Hang Qu, Omid Mashayekhi, David Terei, and Philip Levis. Canary: A Scheduling Architecture for High Performance Cloud Computing. arXiv:1602.01412v1 [cs.DC], 2016.

[18] M. Manivannan, B. Juurlink, and P. Stenstrom. Implications of merging phases on scalability of multi-core architectures. In Proceedings of the International Conference on Parallel Processing (ICPP), pages 622-631, 2011.

[19] Mark D Hill and Michael R Marty. Amdahl's law in the multicore era. Computer, 41(7), 2008.

[20] Hao Che and Minh Nguyen. Amdahl's Law for Multithreaded Multicore Processors. Journal of Parallel and Distributed Computing, 74(10):3056-3069, October 2014. 
[21] Stijn Eyerman and Lieven Eeckhout. Modeling critical sections in Amdahl's Law and its implications for multicore design. In Proceedings of the 37th Annual International Symposium on Computer Architecture, pages 362-370. ACM, 2010.

[22] Gang Ren, Eric Tune, Tipp Moseley, Yixin Shi, Silvius Rus, and Robert Hundt. Google-wide profiling: A continuous profiling infrastructure for data centers. IEEE micro, 30(4):65-79, 2010.

[23] Omid Alipourfard, Hongqiang Harry Liu, Jianshu Chen, Shivaram Venkataraman, Minlan Yu, and Ming Zhang. Cherrypick: Adaptively unearthing the best cloud configurations for big data analytics. In NSDI, volume 2, pages 4-2, 2017.

[24] Janki Bhimani, Ningfang Mi, Miriam Leeser, and Zhengyu Yang. Fim: performance prediction for parallel computation in iterative data processing applications. In Cloud Computing (CLOUD), 2017 IEEE 10th International Conference on, pages 359-366. IEEE, 2017. 